Bien que ce soit une règle de la rédaction du Bulletin, règle imposée par les circonstances, de ne pas faire de place en principe aux sections de la Croix-Rouge dans un pays, nous désirons cependant dire un mot de la Croix-Rouge anglaise en Ecosse, à l'occasion du premier rapport publié par sa section écossaise ${ }^{1}$. Cette dernière est présidée par la duchesse de Montrose, et a le comte de Dalkeith à la tête de son Comité exécutif.

Grâce au zèle de la duchesse de Montrose, qui est l'opposé d'une présidente purement décorative, des comités locaux ont été en 1909 fondés dans presque chaque comté de l'Ecosse. Les efforts du Comité exéculif ont porté sur l'organisation, l'équipement et l'entraînement des corps volontaires, en vue d'en faire des brigades territoriales écossaises de la Croix-Rouge. Une éntente est intervenue, concernant cette formation de personnel, avec la St-Andrew's Ambulance Association.

L'œuvre de la Croix-Rouge étant toute nouvelle pour le public, des assemblées furent tenues pour lui en faire comprendre l'utilité et la beauté. Des brochures de propagande ont été également distribuées largement. Un éveil se produit de tous côtés, à la suite de cette campagne, et des demandes de renseignements affluent de toutes parts. Le secrétaire du Comité exécutif, M. H. S. Barnes (dont les fonctions sont purement honorifiques, honorary secretary) et qui est en même temps secrétaire général de la St-Andrew's Ambulance Association, a donné huit conférences dans des districts divers. Mais le nombre des membres est encore très insuffisant si la branche écossaise veut pouvoir réunir les ressources financières nécessaires.

\title{
FLORENCE NIGHTINGALE
}

Florence Nightingale, la mère des infirmières volontaires, celle à laquelle on a fait en 1908, à l'occasion de ses 88 ans, l'honneur insigne de la recevoir bourgeoise d'honneur de Londres "2, vient de

1 Voy. aux Ouvrages reçus, p. 210.

2 Voy. T. XXXIX, p. 226. 
s'éleindre paisiblement, dans sa quatre-vingt-onzième année, le 13 aoùt 1910.

Née en 1820, à Florence, d'une familie anglaise, elle reçut une instruction soignée el se sentit de bonne heure attirée vers l'étude de la médecine et poussée à consacrer ses journées à visiter les hôpitaux. Elle déplorait déjà alors que les docteurs ne fussent pas assistés par des infirmières plus instruites et plus consciencieuses.

Fn $1845^{1}$, F. Nightingale fit, avec ses parents et sa sœur, de longs voyages en Allemagne, en Italie, en France, en Egypte et en Turquie; elle s'informait partout avec détail des efforts tentés, dans ces divers pays, pour le soin des malades indigents. Elle tint ensuite à faire un apprentissage personnel chez les sœurs de la Charité de Paris, puis à partir de 1849, chez les diaconesses de Kaiserswerth, sur lesquelles elle publia en 1850 une notice en langue anglaise. N'ayant pu réaliser son projet de créer, dans son propre pays, une institution de diaconesses évangéliques, elle consacra, après son retour en Angleterre, son temps, ses forces et ses ressources à la réorganisation d'un asile ou hospice pour les institutrices malades, situé à Jondres (90 Harley street).

Comment ne pas rappeler le magnifique épisode, bien connu d'ailleurs, qui la rendit justement célèbre? C'était en 1854, pendant la guerre de Crimée. Dans les ambulances anglaises, mal organisèes, les blessés mouraient comme des mouches. Un correspondant du Times jeta le cri d'alarme, demandant des femmes dévouées pour soigner les soldats agonisant à l'hôpital de Scutari, Et quand le sous-secrétaire d'Etat, M. Sidney Herbert, songe à Florence Nightingale, elle a déjà écrit qu'elle était prête à partir pour Scutari. Quelques jours après, accompagnée de 37 dames. appartenant comme elle aux classes élevées de la société, elle arrivait à Constantinople.

( Chaque jour, dit un témoin de ces scènes admirables el douloureuses, chaque jour apportait quelques nouvelles complications de misère, toujours surmontées par le génie d'administration de la supérieure; chaque jour apportait son épreuve particulière à celle qui avait pris sur elle le fardeau d'une si immense responsabilité dans un champ inexploré, et avec un état major de son propre sexe à qui tout était également nouveau.

1 Semaine religieuse de Genève, numéro du 20 août 1910. 


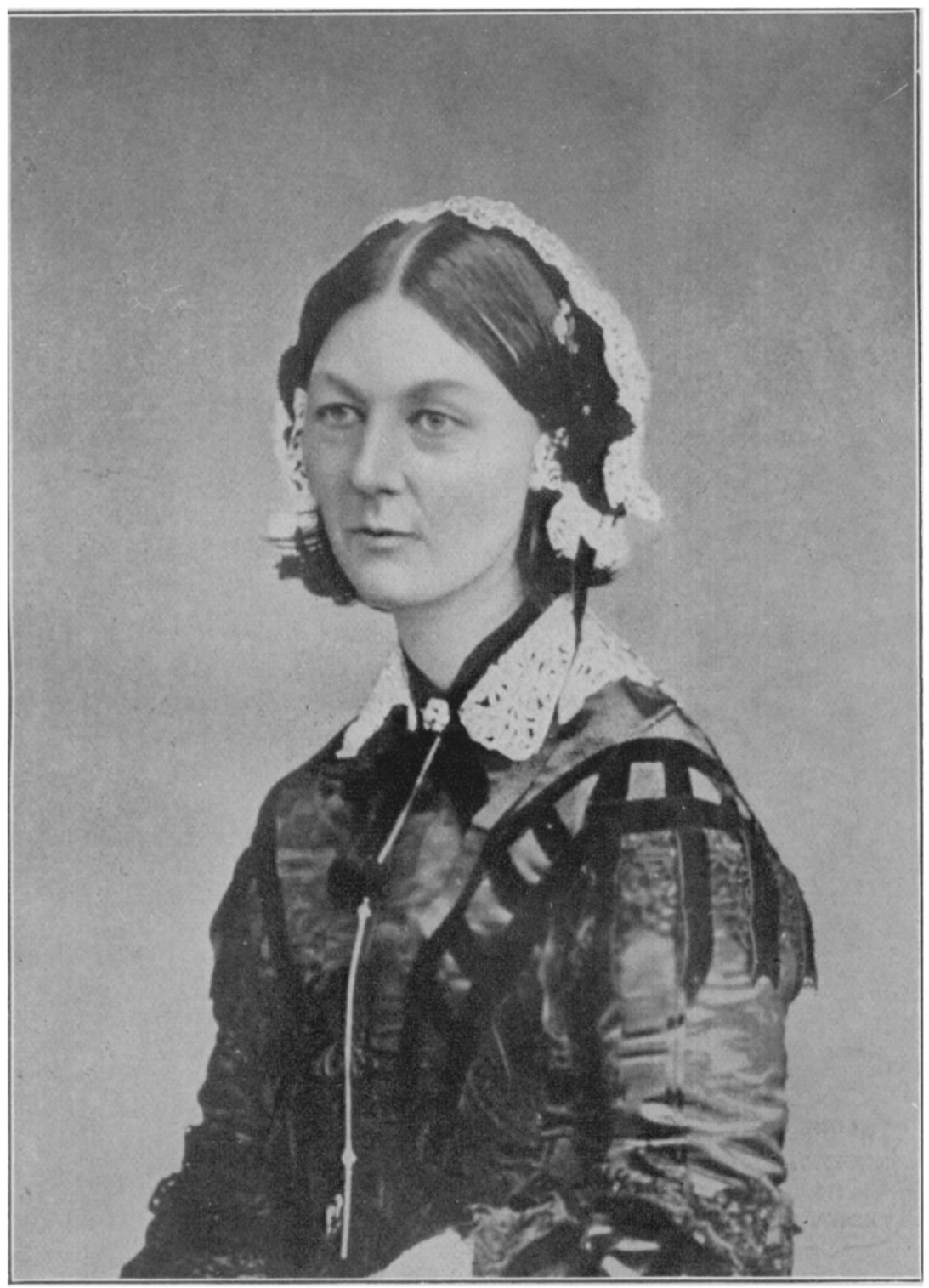

Florence Nightingale

dicidie le $\mathrm{I} 3$ aoilt IOIO 
"On l'a vue souvent à l'arrivée de détachements malades, rester sur pied pendant 24 heures de suite, appropriant les logements, distribuant les vivres, dirigeant les travaux des sceurs, assistant aux plus pénibles opérations lorsque sa présence pouvait fortifier et consoler le patient et passant des heures entières auprès du lit des soldats dans l'agonie du choléra et du typhus.

"L'image de Florence Nightingale parcourant pendant la nuit, la lampe à la main, des dortoirs de plusieurs milles d'étendue, prenant note de l'élat de chaque malade, leur procurant les soulagements les plus nécessaires, ne sera jamais effacée du cœur des hommes, objets ou témoins de sa charité, et la tradition de son dévouement sera à jamais conservée dans l'histoire. »

Tombée malade à la suite de l'épuisement résultant de ses veilles et ses efforts extraordinaires, elle voulut rester malgré la fièvre de Crimée qui l'avait atteinte, et soutenue par son devoir et son intense amour pour les blessés, elle eut la force héroïque de demeurer jusqu'en 1856, jusqu'à la fin, fidèle à son poste et ardente à l'accomplissement de sa tâche. Elle avait réussi à sauver un grand nombre de vies.

Florence Nightingale n'avail pas obligé des ingrats: elle fut récompensée de ses nobles efforts par la reconnaissance et la vénération universelles. Les militaires l'idolâtraient, ils baisaient son ombre quand elle passait dans une chambrée, el, quand elle recouvra la santé après avoir donné des inquiétudes à ses amis, toute l'armée anglaise fut transportée d'allégresse.

Lorsqu'elle revint dans son pays en aoùt 1856 , elle y fut accueillie par d'innombrables témoignages de gratitude. Le sultan lui envoya un bracelet précieux; la reine Victoria lui fit cadeau d'une croix ornée de diamants et l'invita à venir la voir à Balmoral. On fit en son honneur une souscription publique, dont le produit lui fut remis pour une œuvre de son choix. F. Nightingale consacra ce fonds de 45,400 livres sterling, d'abord à la création d'une école d'infirmières qui porte son nom et qui est reliée à un grand hôpita de Londres, l'hôpital St.Thomas, puis à l'entretien d'une école de sages-femmes à l'hòpital de King's College. C'est à ces élablissements que la généreuse amie des malades voua dès lors et jusqu’à la fin de sa vie sa sollicitude empressée. Ses conseils eurent également une influence marquée sur la réorganisalion des ambulances an- 
glaises, sur la création d'écoles de gardes-malades selon son sys. tème. enfin sur la formation des sociétés de la Croix-Rouge ${ }^{1}$.

A ce titre-là seulement, le nom de cette héroüne des premiers secours aux blessés militaires méritait une place honorable dans not re Bulletin.

C'est à l'obligeance du Comité de Washington que nous devons de pouvoir publier son portrait.

\section{JAPON}

\section{LA SOCIÉTÉ JAPONAISE DE 1908-1909²}

Le Bulletin auquel nous empruntons les détails qui vont suivre, débute par un article, dù à la plume du vice-président de la Société, M. le baron T. Ozawa, sur l'importance de la formation d'infirmières ou nurses. La Société japonaise en a, dès longtemps, à l'instar des autres Croix-Rouges, reconnu la nécessité. Elle a en revanche adopté le système, qui n'est pas toujou rs le mode de faire européen, -de former elle-même et au sein de sa propre organisation les infirmières qui lui appartiennent. L'absence d'autres organisations philanthropiques ou religieuses formant desnurses est une des raisons de ce système. L'utilisation des forces féminines dans ce domaine est d'importation toute récente. Les premières infirmières n'étaient que de simples servantes et il a appartenu à la CroixRouge japonaise de relever leur niveau moral et social et de rehausser aux yeux du public la valeur el la noblesse de cette pro. fession de dévouement.

La société fondée, en 1887, par la pripcesse Tarukito, sous le titre de Ladies' Voluntary Nursing Association, a beaucoup contribué à amener ce résultat. Indépendante de la Ciroix-Rouge, elle était composée de dames appartenant en majeure partie à l'aristocratie

1 Semaine religieuse, loc. cit.

${ }^{2}$ D'après le Bulletin $\mathrm{n}^{\circ} 3$ de la Société japonaise. 